\title{
Estabilidad térmica de aleaciones de magnesio Procesadas por Friccion-Agitación (FSP)
}

\author{
Thermal stability of Friction-Stir Processed \\ (FSP) magnesium alloys
}

\author{
Pablo María Delfino ${ }^{1}$, Leonardo Nicolás Tufaro ${ }^{2}$, \\ Hernán Gabriel Svoboda ${ }^{1,3}$
}

\author{
${ }^{1}$ GTSyCM3, INTECIN, Facultad de Ingeniería, Universidad de Buenos Aires/CONICET, Buenos Aires, Buenos Aires, \\ Argenitna \\ e-mail: hsvobod@fi.uba.ar \\ ${ }^{2}$ Grupo de Tecnología de la Soladura, Centro de Mecánica, INTI, San Martín, Buenos Aires, Argentina \\ e-mail: ltufaro@inti.gob.ar; pmdelfino@gmail.com
}

\section{RESUMEN}

La superplasticidad está caracterizada por una extensa deformación plástica previa a la fractura, generalmente a elevada temperatura. En el modo de superplasticidad por estructura fina, el deslizamiento de fronteras de grano es el mecanismo que controla la deformación superplástica. Para lograr esta estructura de grano fino se puede utilizar algún proceso que introduzca una deformación plástica severa, como el Procesamiento por Fricción Agitación (FSP). Sin embargo, se debe tener en cuenta la estabilidad térmica de la estructura obtenida, la cual está asociada a la capacidad que tiene la misma de soportar calentamientos sin que se produzca crecimiento anormal de grano. Por otra parte, el interés por obtener estructuras más livianas en la industria del transporte ha promovido a la incorporación de las aleaciones de magnesio.

El objetivo de este trabajo es analizar la estabilidad térmica de una aleación de magnesio AZ31 procesada por fricción agitación.

A partir de un lingote de AZ31 se obtuvieron chapas de $3 \mathrm{~mm}$ de espesor en condición inicial "as cast". Estas chapas fueron procesadas por fricción agitación bajo condiciones de procesamiento determinadas. Sobre muestras procesadas se realizaron tratamientos térmicos a $300,350,400$ y $450{ }^{\circ} \mathrm{C}$ por un tiempo de 10 , 30 y 60 minutos. Estas 12 condiciones más el material base y el material "como procesado" fueron preparadas para observación metalográfica, obteniéndose el tamaño de grano promedio así como la distribución de tamaño de grano. Asimismo, se determinaron perfiles de microdureza Vickers en cada una de las muestras analizadas. Se evalúa la cinética del crecimiento de grano en función de la temperatura y el tiempo, siendo estos resultados de gran utilidad para el análisis del comportamiento superplástico de estas muestras procesadas en futuros trabajos.

Palabras clave: aleaciones de magnesio, Procesamiento por Fricción-Agitación (FSP), estabilidad térmica, tamaño de grano.

\section{ABSTRACT}

Superplasticity is defined by an extended plastic deformation previous to fracture, generally at high temperatures. In the mode of fine structures superplasticity, grain boundary sliding is the controlling mechanism. To achieve this fine grained structure it could be used any Severe Plastic Deformation (SPD) process like Friction Stir Processing (FSP). Nevertheless, it must be consider the thermal stability of the microstructure produced, which is related to its capacity to resist heating without experiment abnormal grain growth. The use of magnesium alloys has been promoted in the automotive industry in order to reduce weight of the structures.

The aim of this work is to study the thermal stability of the AZ31 friction stir processed microstructure. Sheets of $3 \mathrm{~mm}$ thickness were obtained in as cast condition. These sheets were FSProcessed. Samples of AZ31 FSPed were heat treated to $300,350,400$ and $450{ }^{\circ} \mathrm{C}$, during 10,30 and $60 \mathrm{~min}$. These 12 conditions plus the base material were microstructurally characterized. Vickers microhardness profiles were also measured. Grain growth kinetics was analyzed and related with time and temperature. 
Keywords: Magnesium alloys, Friction-Stir Processing, thermal stability, grain size.

\section{INTRODUCCIÓN}

Debido a la necesidad de disminuir el peso de componentes de la industria del transporte, las aleaciones de magnesio han ido tomando importancia a causa de su baja densidad $(1,7 \mathrm{~g} / \mathrm{cm} 3)$ y al mismo tiempo una buena relación resistencia/peso. La aleación AZ31 ha venido siendo utilizada en el último tiempo para la fabricación de elementos estructurales. Sin embargo, la estructura hexagonal compacta (hcp) del magnesio y sus aleaciones limita la conformabilidad del material.

El refinamiento de grano es un método efectivo para obtener una estructura con propiedades superplásticas, promovido por la activación del mecanismo de deslizamiento de borde de grano [1]. Se requieren típicamente un grano fino con menos de $10 \mu \mathrm{m}$ de diámetro y velocidades de deformación moderadas para activar este mecanismo de deformación de alta temperatura. Debido a que las temperaturas de conformado superplástico se encuentran entre 0,5-0,8 de la temperatura de fusión en grados Kelvin, una microestructura con propiedades superplásticas debe poseer no solo un tamaño de grano fino, sino también una considerable estabilidad térmica a la temperatura de conformado [2].

Una de las formas más comunes para lograr un refinamiento de grano es aplicando alguna técnica de deformación plástica severa (SPD). Este tipo de procesamiento de conformado de metales produce muy altas deformaciones plásticas del material sin cambios significativos en la forma original de la pieza. ECAP (Equal Channel Angular Pressing), HPT (High Pressure Torsion), ARB (Accumulative Roll Bonding) y FSP (Friction Stir Processing) entre otros se han aplicado con esta finalidad en los últimos años [3-7].

El FSP es una técnica derivada de la soldadura por fricción agitación (FSW) que resulta ser una alternativa efectiva para la obtención de una refinamiento de grano con aptitudes superplásticas. A partir de una herramienta no consumible, de forma cilíndrica con un hombro y un pin central. El pin de la herramienta penetra el material a procesar y el calor generado por fricción por el pin y el hombro plastifica el material y la rotación produce la agitación y mezclado. Como consecuencia de la acción de la herramienta sobre el material se generan zonas con diferentes microestructuras, comúnmente denominadas como zona agitada (SZ), zona termo-mecánicamente afectada (TMAZ) y zona afectada por el calor (HAZ) [2,8].

Una estructura de grano refinado obtenido mediante alguna técnica de deformación plástica severa, puede presentar al ser sometida a ciertas condiciones de temperatura y tiempo un crecimiento normal $[3,5]$ o anormal de grano [7], pudiendo definirse una determinada estabilidad térmica de la estructura refinada. Se le atribuyen estos resultados a los parámetros del procesamiento, siendo otro factor de relevancia la condición de partida del material, pudiendo ser en general laminado, extruido, fundido u otra. Sin embargo, es escasa la información disponible respecto de este último aspecto sobre la estabilidad térmica de materiales procesados.

El objetivo del presente trabajo es evaluar la estabilidad térmica de una estructura refinada de una aleación de Mg del tipo AZ31 colada, obtenida mediante FSP.

\section{MATERIALES Y MÉTODOS}

\subsection{MateriaL Base}

En el presente trabajo se estudió una aleación de Mg del tipo AZ31 en condición "como fundida". En la Tabla 1 se muestra la composición química del material empleado expresada en porcentaje en peso (wt\%).

Tabla 1: Composición química del AZ31 estudiado (wt\%).

\begin{tabular}{l|l|l|l|l|l|l}
\hline MATERIAL & Al & Zn & Mn & Fe & Ni & Mg \\
\hline AZ31 & 2,97 & 0,85 & 0,7 & 0,002 & 0,001 & Bal. \\
\hline
\end{tabular}

La composición química del material corresponde a la de la aleación en estudio. En la Figura 1 se muestra una imagen de la microestructura de partida del material. Pueden verse granos equiaxiados de gran tamaño, con presencia de maclas. El tamaño de grano inicial fue de 194 micrones y la microdureza Vickers (HV0,2kg) fue de 45 . 
a
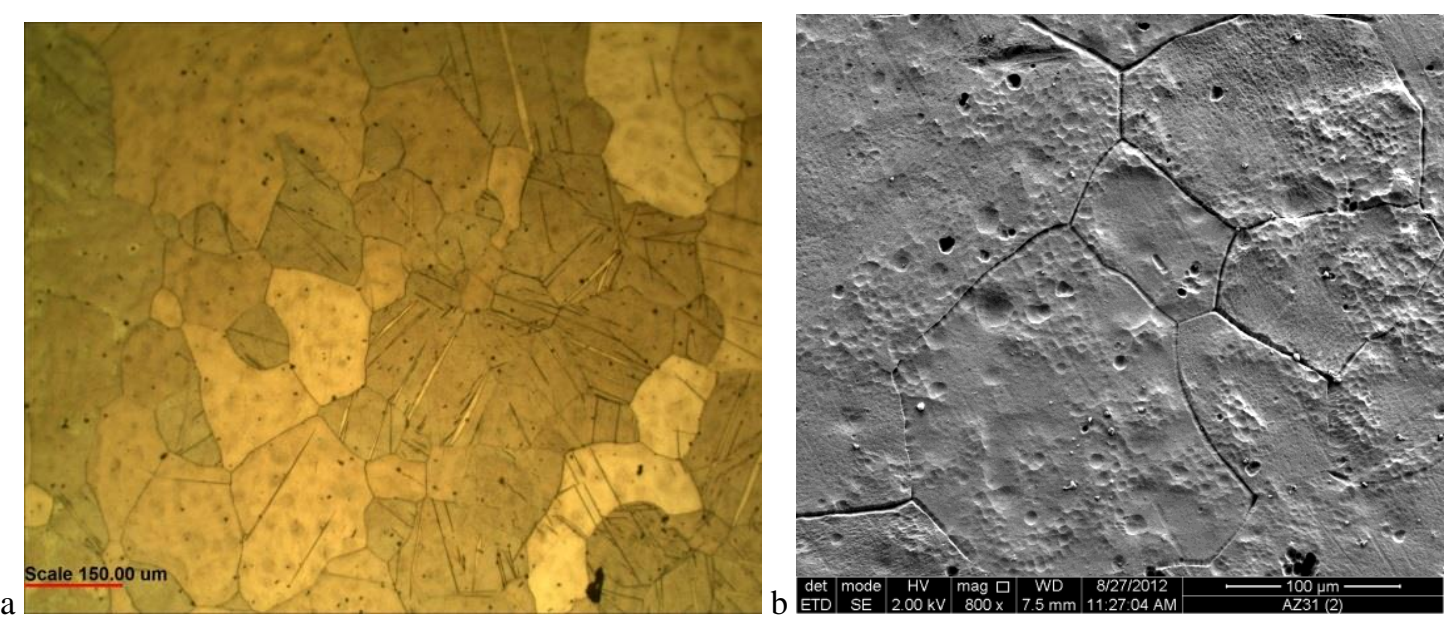

Figura 1: Microestructura inicial del AZ31, a- Microscopía óptica, b- Microscopía electrónica de barrido.

\subsection{Procesamiento FSP}

A partir de un lingote fundido de este material se mecanizaron placas de $150 \times 50 \times 3 \mathrm{~mm}$ para Procesamiento por Fricción-Agitación (FSP). El procesamiento se realizó de acuerdo a los parámetros mostrados en la Tabla 2.

Tabla 2: Parámetros de FSP del AZ31.

\begin{tabular}{l|l|l|l}
\hline MATERIAL & $\begin{array}{l}\text { VEL. ROT. } \\
\text { (RPM) }\end{array}$ & $\begin{array}{l}\text { VEL. AVANCE } \\
\text { (MM/MIN) }\end{array}$ & $\begin{array}{l}\text { ANG. INCL. } \\
\left({ }^{\circ}\right)\end{array}$ \\
\hline $\mathrm{AZ31}$ & 903 & 35 & 1,5 \\
\hline
\end{tabular}

Para esto se empleó una máquina herramienta adaptada para este proceso. La herramienta empleada presentó un hombro cóncavo de $12 \mathrm{~mm}$ de diámetro, un pin cónico con tres caras planas de 2,8 mm de longitud y 3,5 mm de diámetro en la base mayor del cono y fue construida en acero para herramientas del tipo H13.

\subsection{Caracterización de muestra procesada FSP}

A partir de la placa procesada por FSP se extrajeron se realizó la caracterización macro y microestructural sobre un corte transversal que fue preparado para observación metalográfica. Mediante microscopía óptica se analizó la presencia de defectos asociado al FSP y se evaluó la microestructura en las distintas zonas. Particularmente en la zona recristalizada (WN) se llevó a cabo un análisis de tamaño de grano en cinco zonas sobre la línea central del material procesado, equidistantes en el espesor, desde la superficie hasta la base.

Para esto se desarrolló un programa en OCTAVE® que permite analizar imágenes digitales de la microestructura, entregando como resultado la medición de los tamaños de los granos en cada imagen (Figura 2), así como un análisis estadístico de los mismos. A partir de la distribución de tamaños de grano obtenida, se ajustó una función gamma, de la que se obtuvieron las constantes que la definen, para cada una de las zonas analizadas.
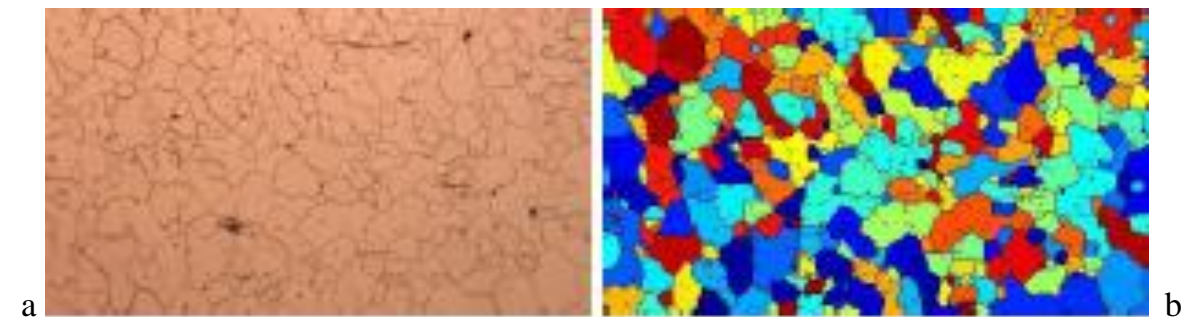

Figura 2: Imagen de entrada (a) y de salida (b) del programa desarrollado para análisis de imágenes. 
Asimismo, se realizó un mapeo de microdureza Vickers (HV0,2 kg) de acuerdo a lo mostrado en la Figura 3, obteniéndose mapas de microdureza de la zona procesada.
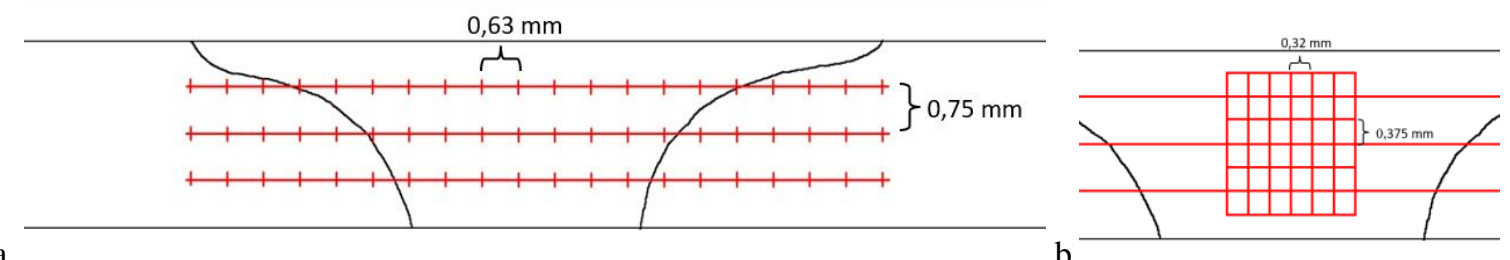

Figura 3: Mapeos de microdureza Vickers en la zona central del WN.

\subsection{Estabilidad Térmica}

A partir de la placa procesada se extrajeron muestras de 20x10x3 mm que involucraban a todo el volumen procesado y se las sometió a distintos tratamientos térmicos en un horno de cámara, a fin de evaluar la estabilidad térmica de la estructura obtenida mediante el FSP. En la Tabla 3 se muestran los distintos tratamientos térmicos realizados.

Tabla 3: Tratamientos térmicos realizados.

\begin{tabular}{l|l|l}
\hline MUESTRA & $\begin{array}{l}\text { TEMPERATURA } \\
\left({ }^{\circ} \mathbf{C}\right)\end{array}$ & $\begin{array}{l}\text { TIEMPO } \\
(\text { MIN })\end{array}$ \\
\hline $300-10$ & 300 & 10 \\
\hline $300-30$ & 300 & 30 \\
\hline $300-60$ & 300 & 60 \\
\hline $350-10$ & 350 & 10 \\
\hline $350-30$ & 350 & 30 \\
\hline $350-60$ & 350 & 60 \\
\hline $400-10$ & 400 & 10 \\
\hline $400-30$ & 400 & 30 \\
\hline $400-60$ & 400 & 60 \\
\hline $450-10$ & 450 & 10 \\
\hline $450-30$ & 450 & 30 \\
\hline $450-60$ & 450 & 60 \\
\hline
\end{tabular}

Sobre las muestras tratadas térmicamente se realizó una caracterización microestructural, analizando el tamaño de grano en cinco zonas de forma similar a lo descripto en el apartado 2.3. Asimismo, para cada caso se realizó el análisis de microdureza de acuerdo a lo descripto en el mismo apartado.

\section{RESULTADOS}

\subsection{Caracterización de muestra procesada por FSP}

En la Figura 4 se muestran una macrografía del corte transversal de la probeta procesada.

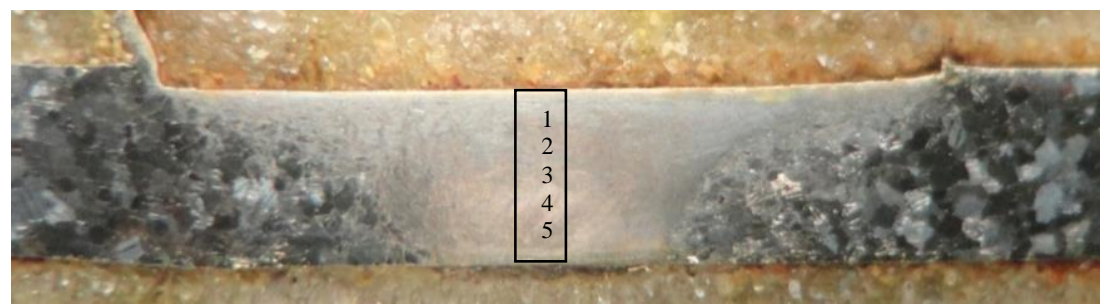

Figura 4: Macrografía de la muestra procesada por FSP. 
Puede verse la zona procesada con la forma caracterísitca asociada a la herramienta. Asimismo se observa la ausencia de defectos y la reducción del tamaño de grano en la zona agitada (WN), respecto al tamaño de grano original. Sobre la linea central de la WN se identifican las 5 zonas donde se caracterizó la microestructura en las probetas tratadas térmicamente y se determinó el tamaño de grano.

En la Figura 5a se muestra una micrografía de la zona central del material procesado. En la Figura $6 \mathrm{~b}$ se muestra el histograma de tamaños de grano obtenido del análisis realizado de la probeta procesada en la zona de la línea central del cordón.
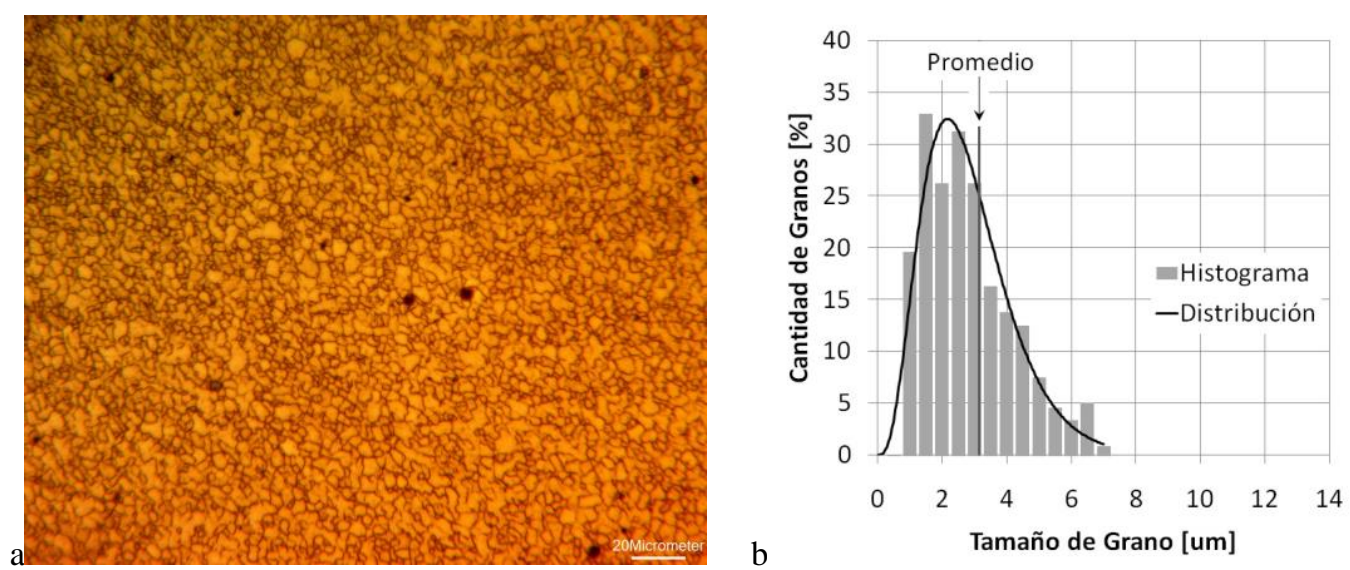

Figura 5: a-Micrografía de la muestra procesada por FSP, b-Histograma de tamaños de grano.

El tamaño de grano promedio determinado del análisis estadístico de los granos fue de 3,1 $\mu$ m, mientras que la moda de la función de ajuste fue de $2,2 \mu \mathrm{m}$ y la varianza fue de $1,88 \mu \mathrm{m}^{2}$. Puede verse que se obtuvo un fuerte refinamiento respecto del tamaño de grano inicial (194 micrones).

En la Figura 6 se muestran los mapas de dureza obtenidos para la probeta procesada.

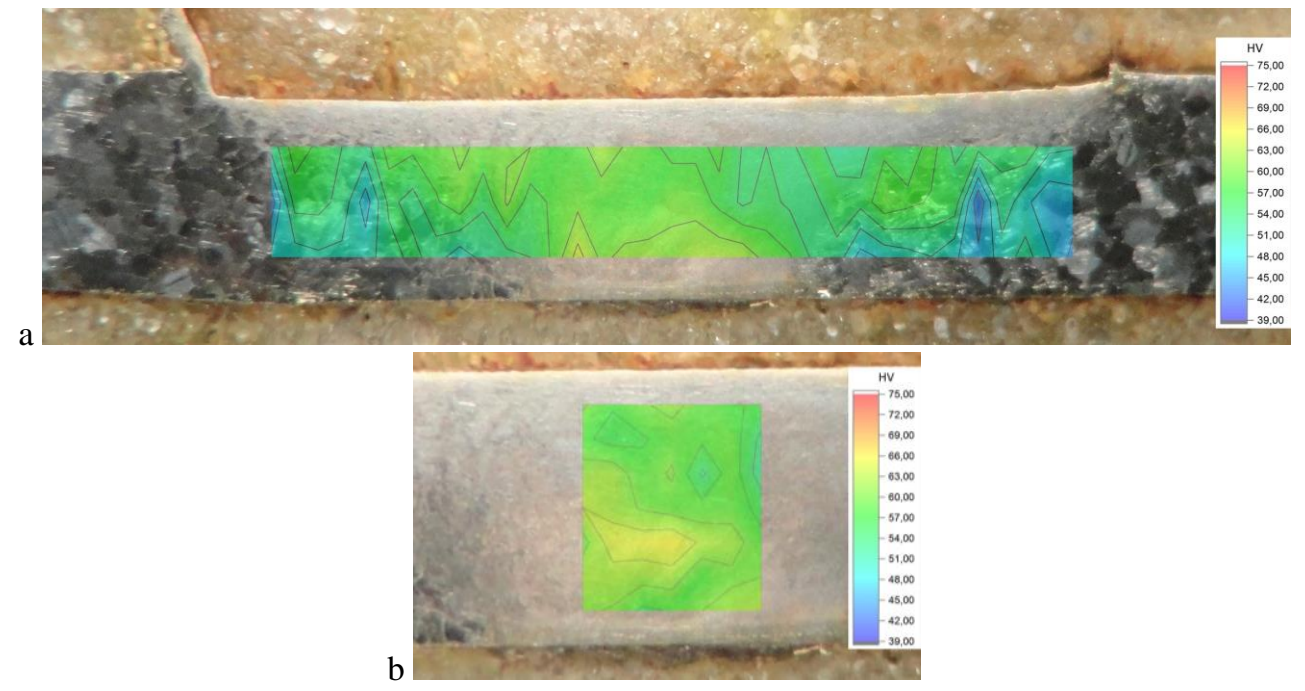

Figura 6: Mapas de microdureza HV del WN de la probeta "como procesada", a- general, b- zona central.

Puede verse que en la zona agitada se tiene un aumento de la dureza respecto de la del material base. Esto estaría asociado el refinamiento de grano observado. En particular en la zona central del WN se observan los mayores valores de dureza que alcanzan los 65-70 HV.

\subsection{Estabilidad térmica}

En las Figuras 7, 8, 9 y 10 se pueden ver las micrografías correspondientes a las muestras tratadas térmicamente a $300,350,400 \mathrm{y} 450^{\circ} \mathrm{C}$, respectivamente, para los tres tiempos de permanencia en el horno. 

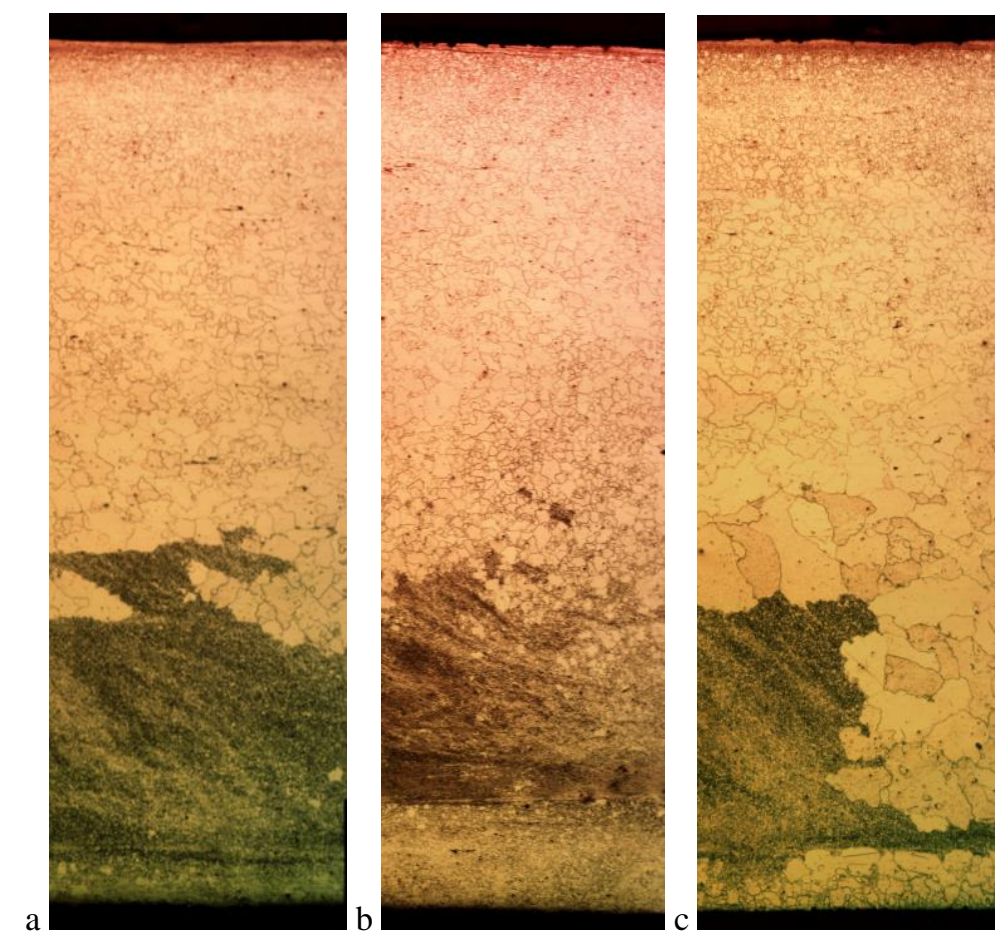

Figura 7: Micrografías de la zona central de las muestras tratadas térmicamente a $300^{\circ} \mathrm{C}$ : a- 10 ., b- 30 y c- 60 min.
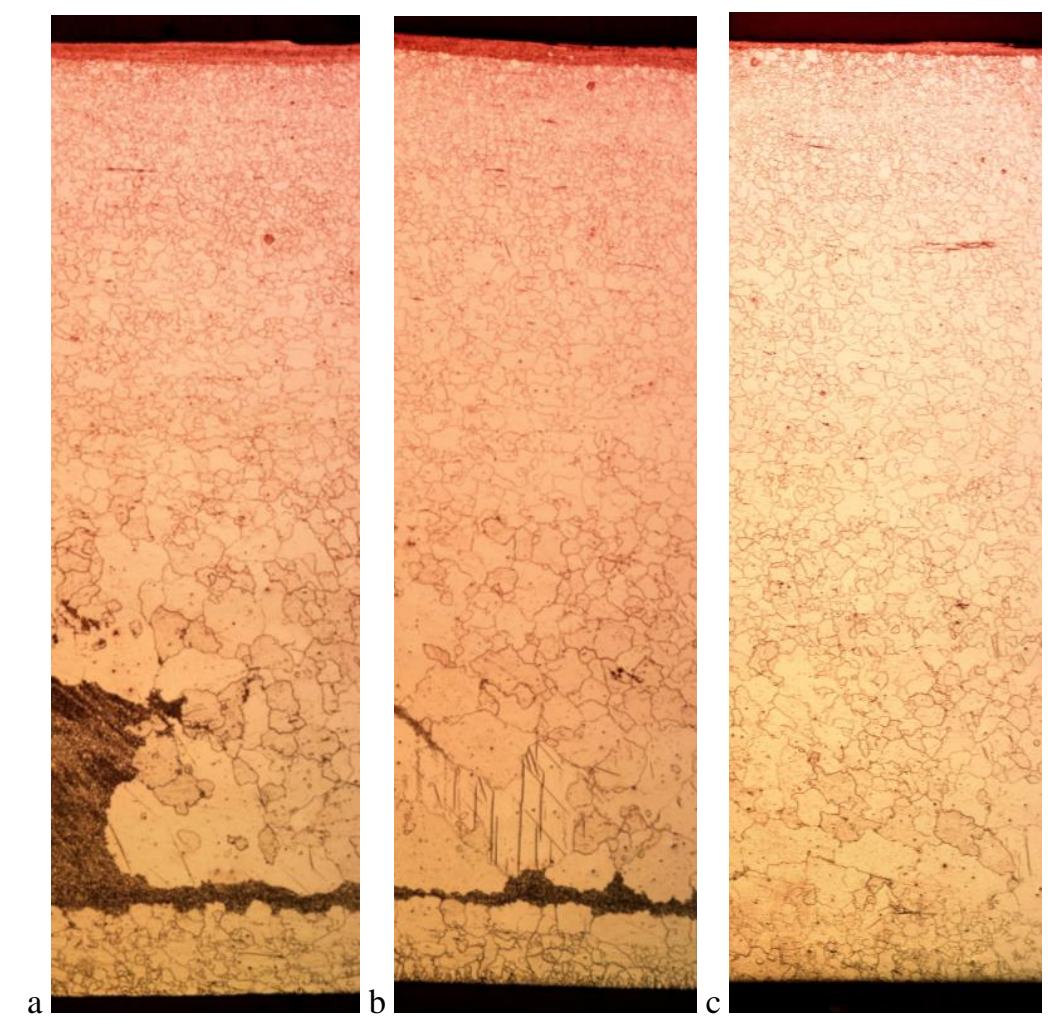

Figura 8: Micrografías de la zona central de las muestras procesadas y tratadas térmicamente a $350^{\circ} \mathrm{C}$ durante: a- 10 min., b-30 min y c-60 min. 

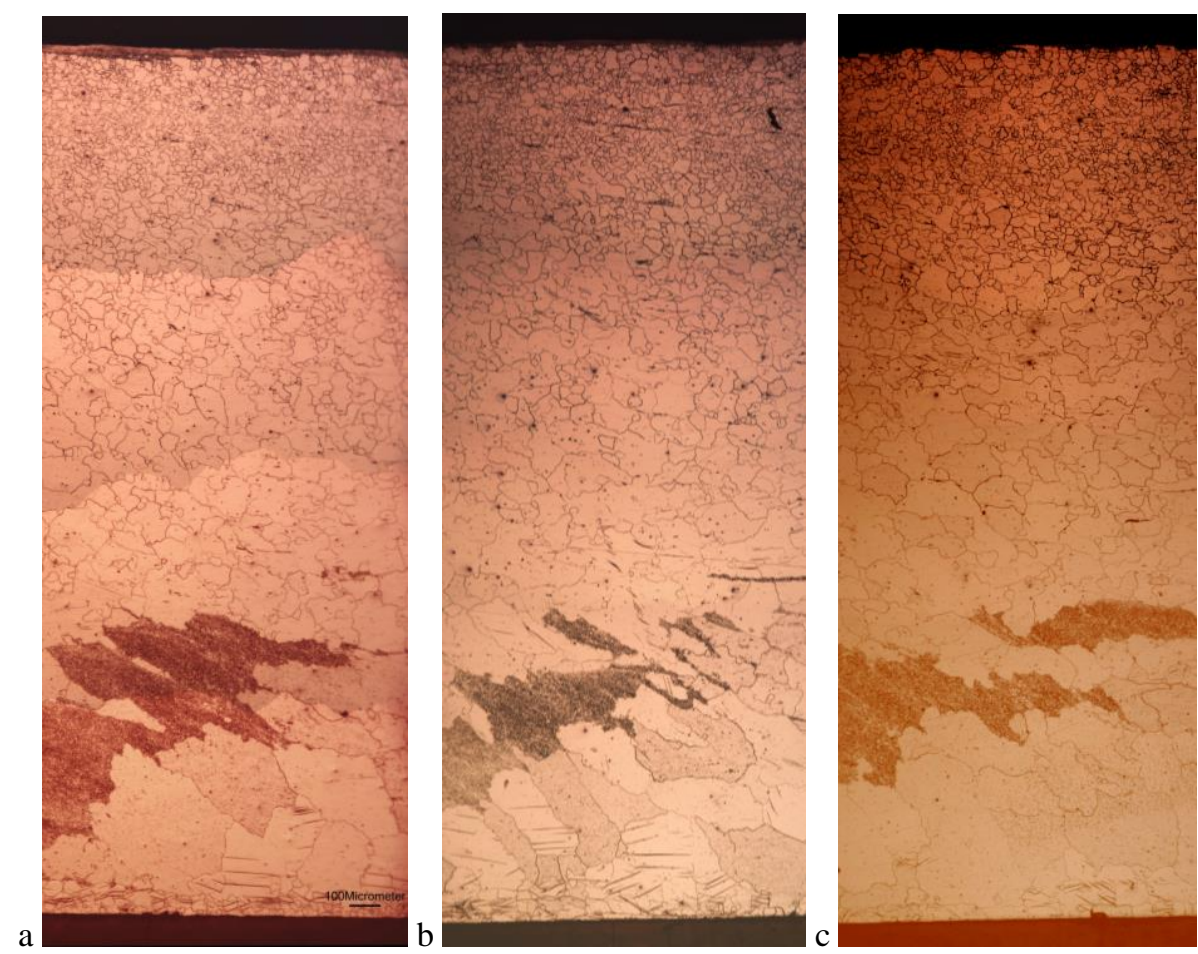

Figura 9: Micrografías de la zona central de las muestras procesadas y tratadas térmicamente a $400^{\circ} \mathrm{C}$ durante: a- 10 min., b-30 min y c-60 min.
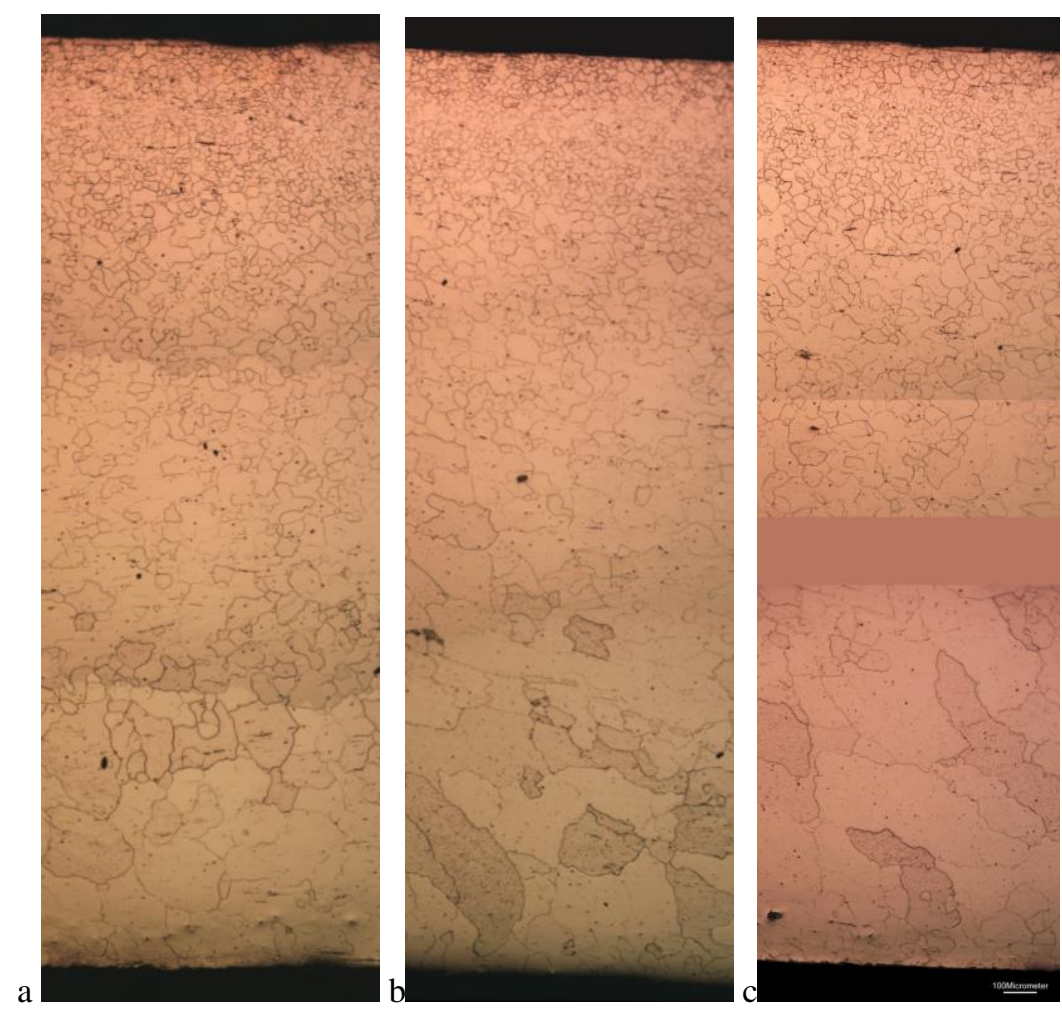

Figura 10: Micrografías de la zona central de las muestras procesadas y tratadas térmicamente a $450^{\circ} \mathrm{C}$ durante: a- 10 min., b-30 min y c-60 min.

Puede verse que se tiene un comportamiento diferenciado de acuerdo a la zona que se considere. La parte superior del espesor, donde se tiene una mayor incidencia del hombro de la herramienta, presenta una mayor estabilidad, observándose un crecimiento normal de grano. En la parte inferior, donde la deformación 
está asociada a la acción del pin, para las menores temperaturas $\left(330,350\right.$ y $\left.400^{\circ} \mathrm{C}\right)$ en los tres tiempos se observa la existencia de una zona con el grano refinado que mantiene el tamaño generado durante el procesamiento, coexistiendo con material que experimenta un crecimiento anormal de grano, alcanzando tamaños de grano del orden o superiores a los del material en la condición inicial. Para $450^{\circ} \mathrm{C}$ no se observa la presencia de zona refinada en la parte inferior del espesor, presentándose solo material que experimentó crecimiento anormal de grano. La medición del tamaño de grano en las zonas 4 y 5 correspondientes a la parte inferior del espesor se vio dificultada debido a la existencia de un tamaño de grano dúplex, como se observa en las figuras 7 a 10.

\subsection{Microdureza}

En las figuras 11 a 14 se observan los mapas de dureza de la zona central de las muestras procesadas y tratadas térmicamente a $300,350,400$ y $450^{\circ} \mathrm{C}$, para 10,30 y 60 min de permanencia.
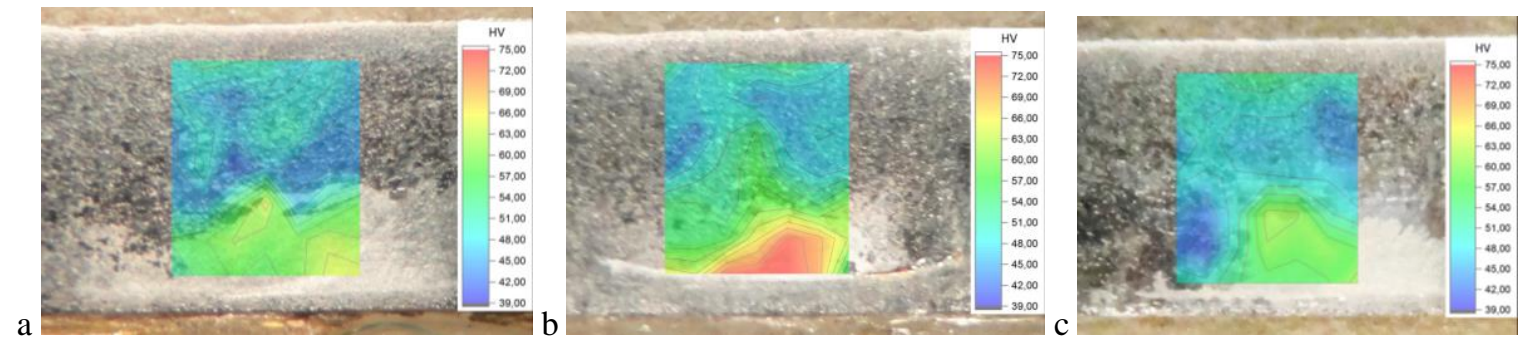

Figura 11: Macrografías y mapas de microdureza Vickers de las muestras procesadas y tratadas térmicamente a $300^{\circ} \mathrm{C}$ durante: a- 10 min., b-30 min y c-60 min.
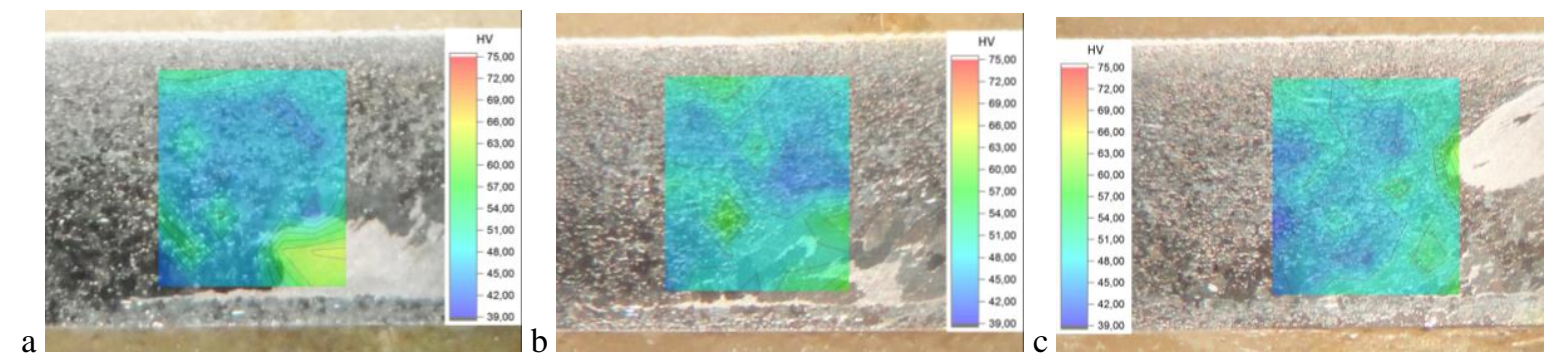

Figura 12: Macrografías y mapas de microdureza Vickers de las muestras procesadas y tratadas térmicamente a $350^{\circ} \mathrm{C}$ durante: a- 10 min., b-30 min y c-60 min.
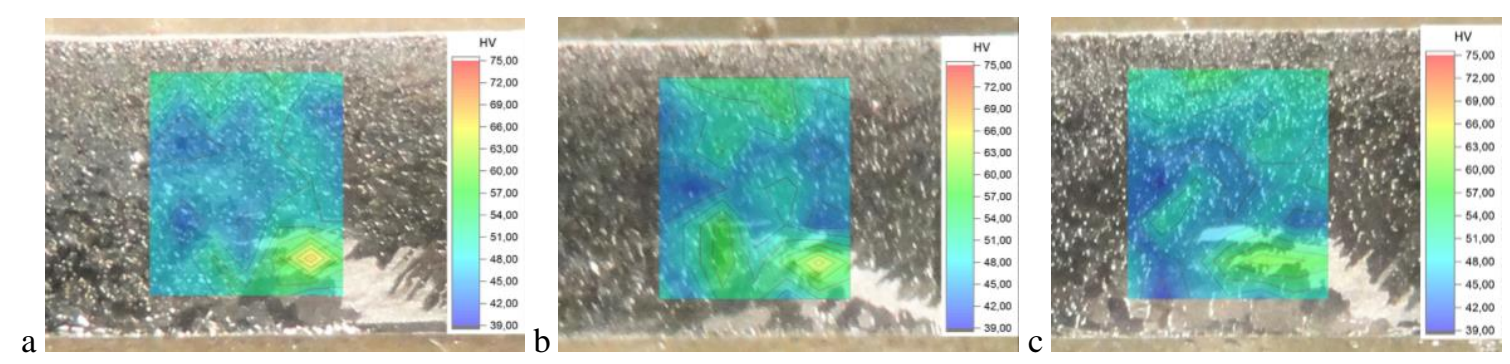

Figura 13: Macrografías y mapas de microdureza Vickers de las muestras procesadas y tratadas térmicamente a $400^{\circ} \mathrm{C}$ durante: a- 10 min., b-30 min y c-60 min. 


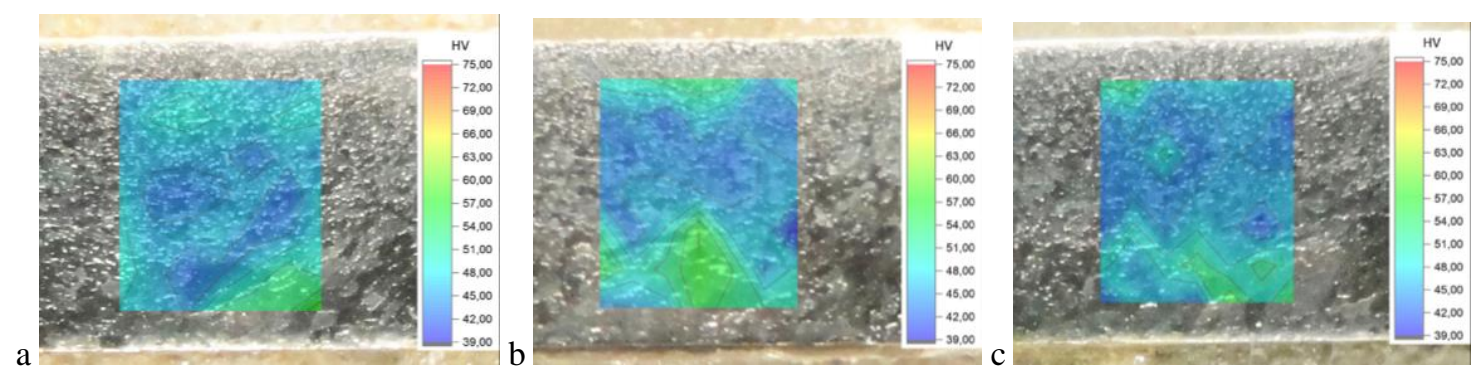

Figura 14: Macrografías y mapas de microdureza Vickers de las muestras procesadas y tratadas térmicamente a $450^{\circ} \mathrm{C}$ durante: a- 10 min., b-30 min y c-60 min.

En todos los casos puede verse que la dureza en la zona inferior de la zona procesada en general el mayor, asociada al mantenimiento de una zona refinada en ese sector. Al perderse esa característica, los valores de dureza disminuyen. A su vez, en la parte superior también se detectaron valores de dureza algo mayores, también vinculados a un crecimiento más lento del tamaño de grano, con la temperatura y el tiempo. En general los valores medidos fueron menores que los obtenidos en la muestra procesada, debido al crecimiento del tamaño de grano.

\section{DISCUSIÓN}

En la Figura 15 se muestran los tamaños de grano resultantes para las distintas muestras tratadas térmicamente a diferentes temperaturas y tiempos, medidos en las zonas 1,2 y 3.

Zona 1

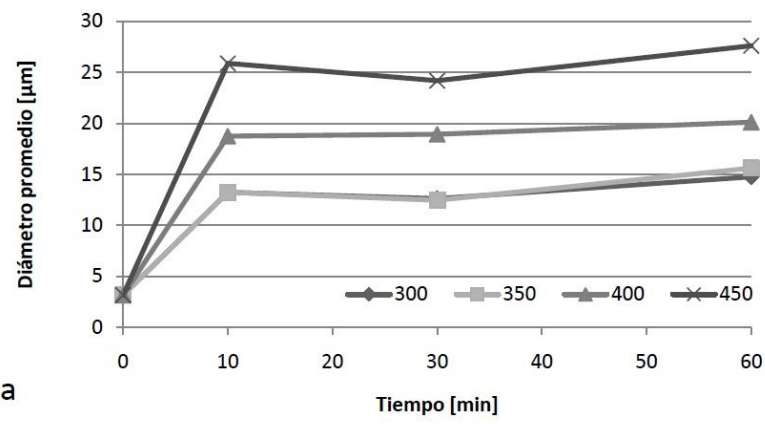

Zona 2

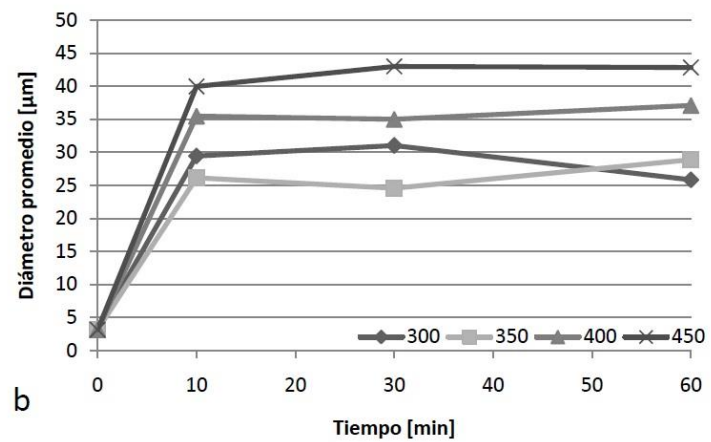

Zona 3

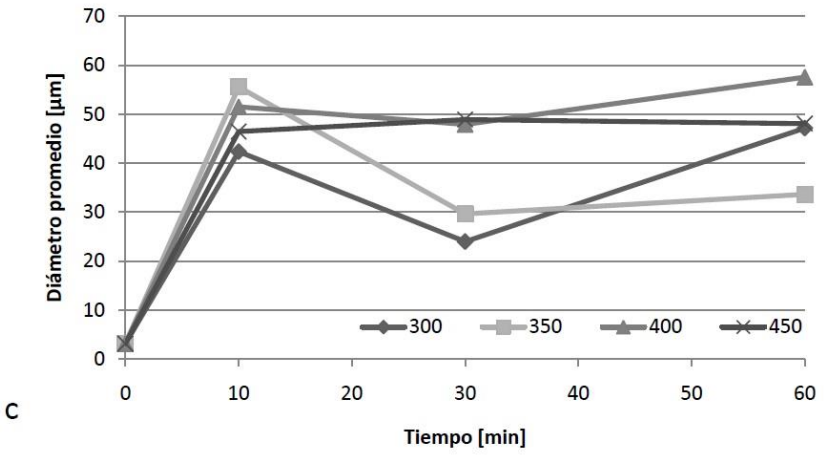

Figura 15: Tamaño de grano en función del tiempo de permanencia a las distintas temperaturas, para: a- Zona 1., b- Zona 2 y c- Zona 3.

Puede verse una evolución dispar del tamaño de grano con la temperatura y el tempo de permanencia, de acuerdo a la zona que se considere. Esto estaría asociado a la diferente historia termomecánica de cada zona. De acuerdo a la zona que se trate dentro de la WN, se tiene diferente nivel de deformación, velocidad de deformación y temperatura [2] lo que generará una diferente estabilidad térmica de la estructura obtenida. 
Asimismo, las condiciones de procesamiento, la geometría de la herramienta y otros aspectos operativos, también tendrán efecto sobre la estructura obtenida luego del procesado. Además la condición de partida también influirá en este sentido. Estos resultados son consistentes con los reportados en bibliografía para este material procesado mediante diversas técnicas de SPD [4-6]. Sin embargo, los tamaños de grano alcanzados y la estabilidad térmica asociada de la estructura en este caso es algo menor que para otros casos donde la condición de partida no es "como fundida" [4-6]. Una etapa de deformación previa a la aplicación de una técnica de SPD evidencia una mayor estabilidad térmica. Esto estaría asociado a la modificación de la estructura de bordes de grano de partida. En este sentido, estudios de bordes y texturas mediante EBSD para esta condición inicial podría aportar más información para la mejor comprensión de los fenómenos que limitan la estabilidad térmica de una estructura procesada que parte de una condición "como fundida".

Puede verse que a medida que aumenta la distancia a la superficie de la probeta, el tamaño de grano alcanzado luego de una dada temperatura y tiempo aumenta. Asimismo, en general se observa que luego de 10 min el tamaño de grano no experimenta un crecimiento adicional significativo. A su vez, en general el tamaño de grano aumenta con la temperatura.

Por otro lado, se observa cierta tendencia donde para 30 min de permanencia, en distintas temperaturas y zonas analizadas, el tamaño de grano presenta una leve disminución. Este aspecto deberá ser analizado con más detalle en futuros trabajos, dado que no es un resultado esperable, ni ha sido reportado previamente.

El problema de la estabilidad térmica de la microestructura es un tema de relevancia dado que define la posibilidad de que el material en esa condición microestructural pueda experimentar deformaciones superplásticas.

En la Figura 16 se muestra la evolución de la microdureza Vickers con el tamaño de grano medido para todas las muestras analizadas.

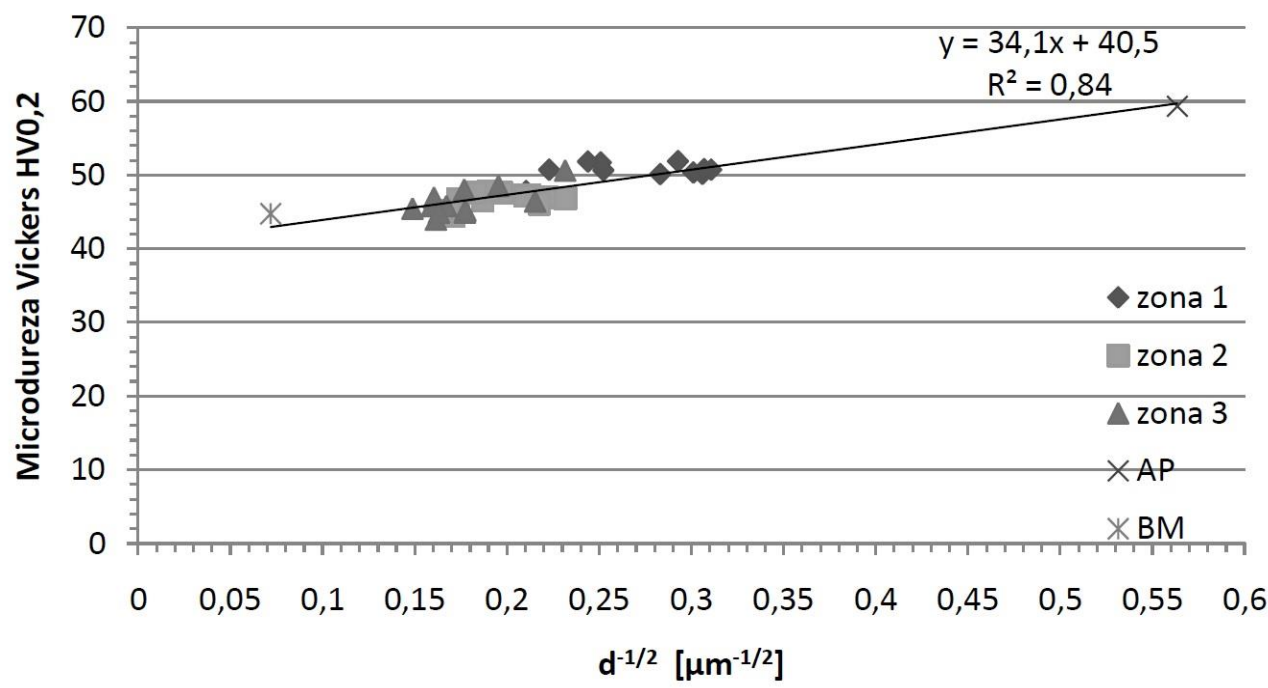

Figura 16: Microdureza Vickers en función de la inversa la raíz del tamaño de grano.

Puede verse que se obtiene una buena correlación entre todos los valores de tamaño de grano medidos y sus correspondientes durezas promedio, considerando las distintas condiciones. Esto indicaría que el mecanismo que controla las propiedades mecánicas en este material sería el de endurecimiento por afino del tamaño de grano. Se observa que la correlación obtenida es buena, con un coeficiente $\mathrm{R}^{2}$ de 0,84 .

Los valores de las constantes obtenidas para la ecuación de Hall-Petch son consistentes con los reportados en la literatura para el mismo material procesado mediante técnicas de SPD $[6,8,9]$.

\section{CONCLUSIONES}

En el presente trabajo se procesó mediante FSP una aleación de magnesio del tipo AZ31, en condición de partida "como fundido", y se evaluó posteriormente la estabilidad térmica de la estructura procesada a distintas temperaturas $\left(300,350,400\right.$ y $\left.450^{\circ} \mathrm{C}\right)$ y tiempos $(10,30$ y $60 \mathrm{~min})$ de permanencia.

Se desarrollaron herramientas para el análisis de tamaños de grano y se realizaron mapas de microdureza Vickers de las distintas zonas de la WN.

Con el procesamiento FSP se logró un fuerte refinamiento de grano desde 194 hasta alrededor de 3 micrones y no se observaron la presencia de defectos. Desde un punto de vista de la aplicación resulta viable 
procesar el material "como fundido" evitándose los productos elaborados de mayor costo.

Luego de la aplicación de los ciclos térmicos, se observó una respuesta dispar de acuerdo a la sección de la WN considerada. En la parte superior, cercana a la acción del hombro de la herramienta FSP, se tuvo una mayor estabilidad y se observó un crecimiento normal de grano. En la zona inferior del WN, donde actúa el pin de la herramienta para bajas temperaturas se mantuvo la estructura refinada coexistiendo con zonas de crecimiento anormal de grano (CAG) y para las mayores temperaturas se observó una fuerte CAG. Estas observaciones están asociadas a la fuerte dependencia de la historia termomecánica del material en relación a la estabilidad térmica de la estructura. La condición de partida "como fundida" limitaría dicha estabilidad, la cual también se vería afectada por las condiciones de procesamiento. Estudios futuros de bordes mediante EBSD podrían aportar a la mejor comprensión de los mecanismos actuantes.

Se obtuvo una buena correlación entre los valores de tamaño de grano y de microdureza relevados mediante la ecuación de Hall-Petch, que indica que el mecanismo de refinamiento de grano es el que controla las propiedades mecánicas del material.

\section{AGRADECIMIENTOS}

Los autores de este trabajo desean agradecer a la Universidad de Buenos Aires, por el apoyo económico para el desarrollo del presente trabajo y a INTI Mecánica por los análisis químicos y las microscopía electrónica de barrido.

\section{BIBLIOGRAFÍA}

[1] SHERBY, Oleg D. "Advances in superplasticity and in superplastic materials" ISIJ International 29, no. 8 pp 698-716, 1989.

[2] MISHRA R. S., MAHONEY, M. W. "Friction Stir Processing”, In: Mishra R. S., Mahoney, M. W. (eds), Friction Stir Welding and Processing, 1 ed., chapter 14, Ohio, USA, ASM International, 2007.

[3] YOUNG, J. P., ASKARI, H., HOVANSKI, Y., et al., "Thermal microstructural stability of AZ31 magnesium after severe plastic deformation", Materials Characterization, n. 101, pp.9-19, 2015.

[4] EDDAHBI, M., DEL VALLE, J.A., PÉREZ-PRADO, M.T. and RUANO, O.A. "Comparison of the microstructure and thermal stability of an AZ31 alloy processed by ECAP and large strain hot rolling”, Materials Science and Engineering: A, n.410, pp.308-311, 2005.

[5] MIAO, Q., HU, L., WANG, X. and WANG, E. "Grain growth kinetics of a fine-grained AZ31 magnesium alloy produced by hot rolling", Journal of Alloys and Compounds, n. 493, v.1, pp.87-90, 2010.

[6] STRÁSKÁ, J., JANEČEK, M., ČÍŽEK, J., et al., "Microstructure stability of ultra-fine grained magnesium alloy AZ31 processed by extrusion and equal-channel angular pressing (EX-ECAP)", Materials Characterization, n. 94, pp.69-79, 2014.

[7] SRINIVASARAO, B., VALLE, J.D., RUANO, O.A., et al., "Influence of thermomechanical processing on the grain size, texture and mechanical properties of Mg-Al alloys", Disponível em: 2012. https://core.ac.uk/download/pdf/36054959.pdf

[8] DORBANE, A., AYOUB, G., MANSOOR, B., et al., "Microstructural observations and tensile fracture behavior of FSW twin roll cast AZ31 Mg sheets", Materials Science and Engineering: A, n.649, pp.190-200, 2016.

[9] KIM, H. K., KIM, W. J. "Microstructural instability and strength of an AZ31 Mg alloy after severe plastic deformation", Materials Science and Engineering: A, vol. 385, n. 1, pp. 300-308, 2004. 